\title{
Efficacy and safety of gemcitabine monotherapy in an elderly patient with penile metastasis from bladder carcinoma: a case report
}

\author{
Masashi Matsushima $\cdot$ Eiji Kikuchi • \\ Yohei Masugi $\cdot$ Hirotaka Akita $\cdot$ Akira Miyajima • \\ Masahiro Jinzaki $\cdot$ Mototsugu Oya
}

Received: 8 December 2012/Accepted: 30 March 2013/Published online: 13 April 2013

(C) The Japan Society of Clinical Oncology 2013

\begin{abstract}
We describe the case of an 85-year-old Japanese male with invasive urothelial carcinoma of the bladder treated with radiotherapy and the significant effects of gemcitabine monotherapy on subsequent metastases to the lungs, L5 vertebral body, and penis. The lung and L5 vertebral body metastases were diagnosed 3 months after radiotherapy and were treated with one course of gemcitabine and cisplatin chemotherapy. As the patient was unable to continue the chemotherapy, the chemotherapy was switched to two further courses of gemcitabine monotherapy, and he showed a significant response to the metastasis. The patient's condition was good until he noticed a penile nodule that appeared 2.5 years after the radiotherapy. The penile tumor was pathologically confirmed as metastatic urothelial carcinoma. The patient received one additional course of gemcitabine monotherapy. At a 1-year follow-up examination, he was still alive and had responded completely to the treatment for the penile metastasis. Metastatic involvement of the penis is an exceptionally rare condition. It is important to consider the balance between the tolerability and efficacy of chemotherapy and that gemcitabine monotherapy might be
\end{abstract}

M. Matsushima · E. Kikuchi $(\bowtie) \cdot$ A. Miyajima $\cdot$ M. Oya Department of Urology, Keio University School of Medicine, 35 Shinanomachi, Shinjuku-ku, Tokyo 160-8582, Japan e-mail: eiji-k@kb3.so-net.ne.jp

Y. Masugi

Department of Pathology, Keio University School of Medicine, Tokyo, Japan

H. Akita · M. Jinzaki

Department of Radiology, Keio University School of Medicine, Tokyo, Japan acceptable in an elderly patient with metastatic urothelial carcinoma who is still in good physical condition. The findings in this case suggest that gemcitabine monotherapy may be one possible candidate for the treatment of metastatic urothelial carcinoma, even in patients aged 80 years or older.

Keywords Penile metastases - Bladder cancer . Gemcitabine $\cdot$ Urothelial carcinoma $\cdot$ Elderly patient

\section{Introduction}

In Japan in 2000, bladder cancer was the 9th most commonly diagnosed cancer in men and the 17th most frequently diagnosed cancer in women [1]. Most cancer patients are elderly, and elderly patients bear the greatest burden of cancer worldwide. The proportion of people over 65 years old is increasing rapidly in Japan. Accordingly, there is an expected rise in the need to treat elderly patients presenting with metastatic urothelial carcinoma (UC), since the median age of patients with this disease is $>70$ years. However, a standard of care for elderly patients with metastatic UC has not yet been established.

Gemcitabine (GEM) is the key chemotherapeutic drug for the treatment of unresectable UC. GEM was approved for UC in Japan in 2008 and will soon be widely used. When used as a single agent in previously untreated patients with metastatic bladder cancer, GEM was found to induce response rates of about $30 \%$ [2]. The toxicity was generally mild, with approximately $25 \%$ of patients experiencing grade 3 neutropenia that was rapidly reversible [2]. However, no reports have evaluated the efficacy and safety of GEM monotherapy for elderly patients with metastatic UC. 


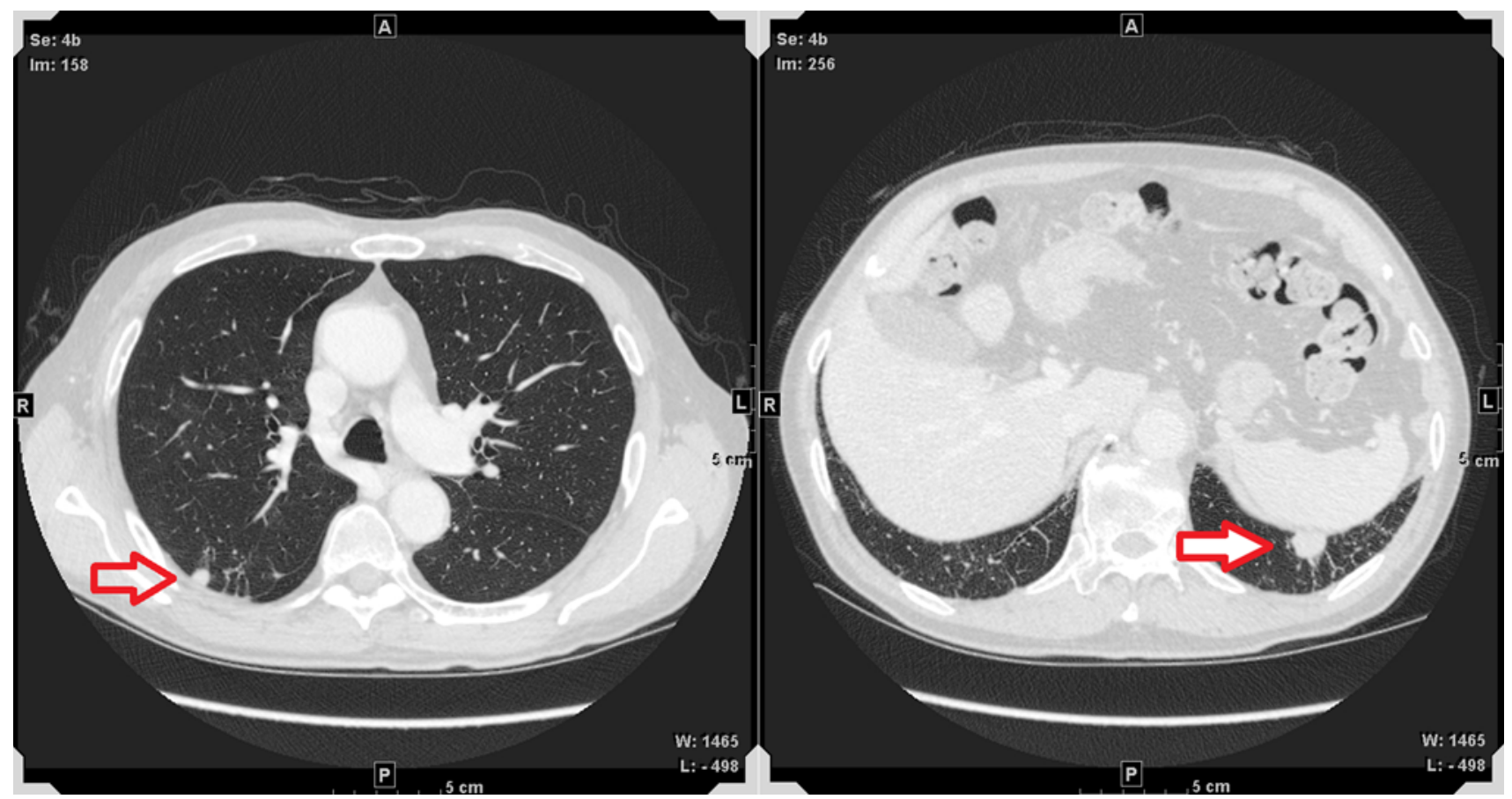

Fig. 1 Chest computed tomography image 3 months after radiotherapy for invasive bladder cancer. A computed tomography examination revealed multiple lung metastases with more than 5 nodules of $<15 \mathrm{~mm}$ in size (arrow)

Metastatic involvement of the penis is an exceptionally rare condition. The first case of penile metastasis was reported by Eberth in 1870 [3]. To date, approximately 370 cases of penile metastasis have been reported worldwide $[4,5]$. Over $70 \%$ of penile metastases have their origin in the genitourinary tract, arising most often from the bladder and prostate [6].

Here, we present the case of an 85-year-old patient with metastatic bladder cancer who was effectively and safely treated with GEM monotherapy. He also had penile metastasis during follow-up.

\section{Case report}

An 85-year-old man was referred to our hospital with muscle-invasive bladder cancer. He had previously undergone transurethral resection of a bladder tumor at another institution, and the pathological examination revealed muscle-invasive urothelial cancer (UC, pT2, grade 3). A computed tomography (CT) examination had revealed no evidence of metastases (clinical stage: T2N0M0). A physical examination did not reveal any unusual findings, and the results of a laboratory evaluation were within the normal limits. The patient underwent 60 Gy of external beam radiotherapy (EBRT) for the locally advanced invasive bladder cancer. Three months after the EBRT, a CT examination revealed multiple lung metastases with more than 5 nodules of $<15 \mathrm{~mm}$ in size (Fig. 1), and a magnetic resonance imaging (MRI) examination revealed L5 vertebral body metastases (Fig. 2).

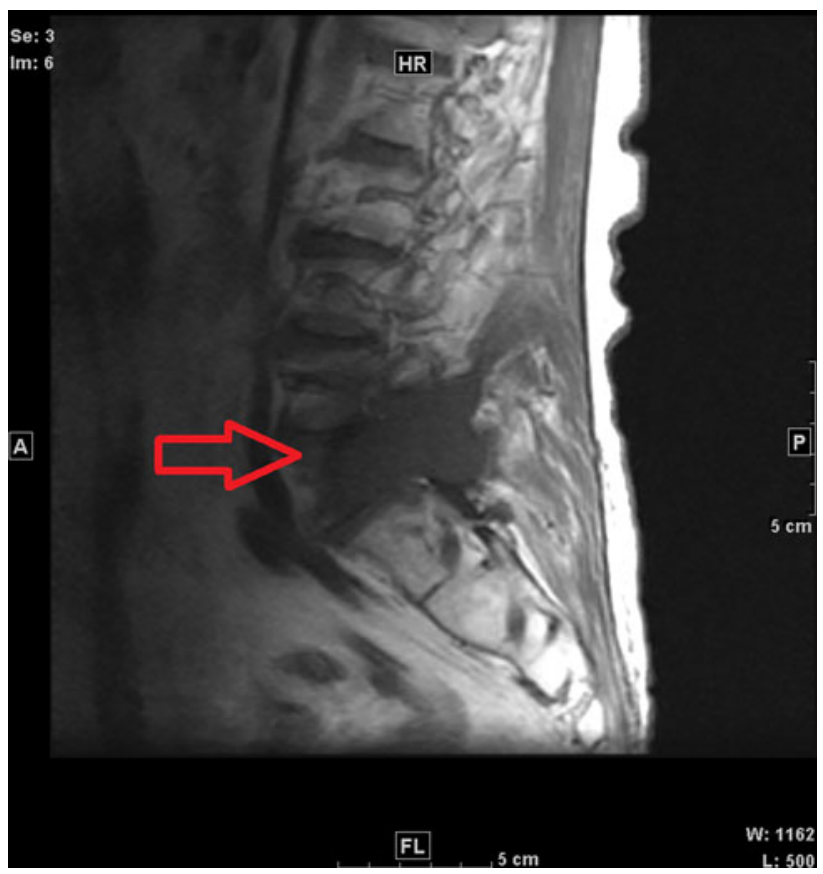

Fig. 2 Magnetic resonance image of lumbar vertebral body 3 months after radiotherapy for invasive bladder cancer. Magnetic resonance imaging revealed L5 vertebral body metastasis (arrow) 


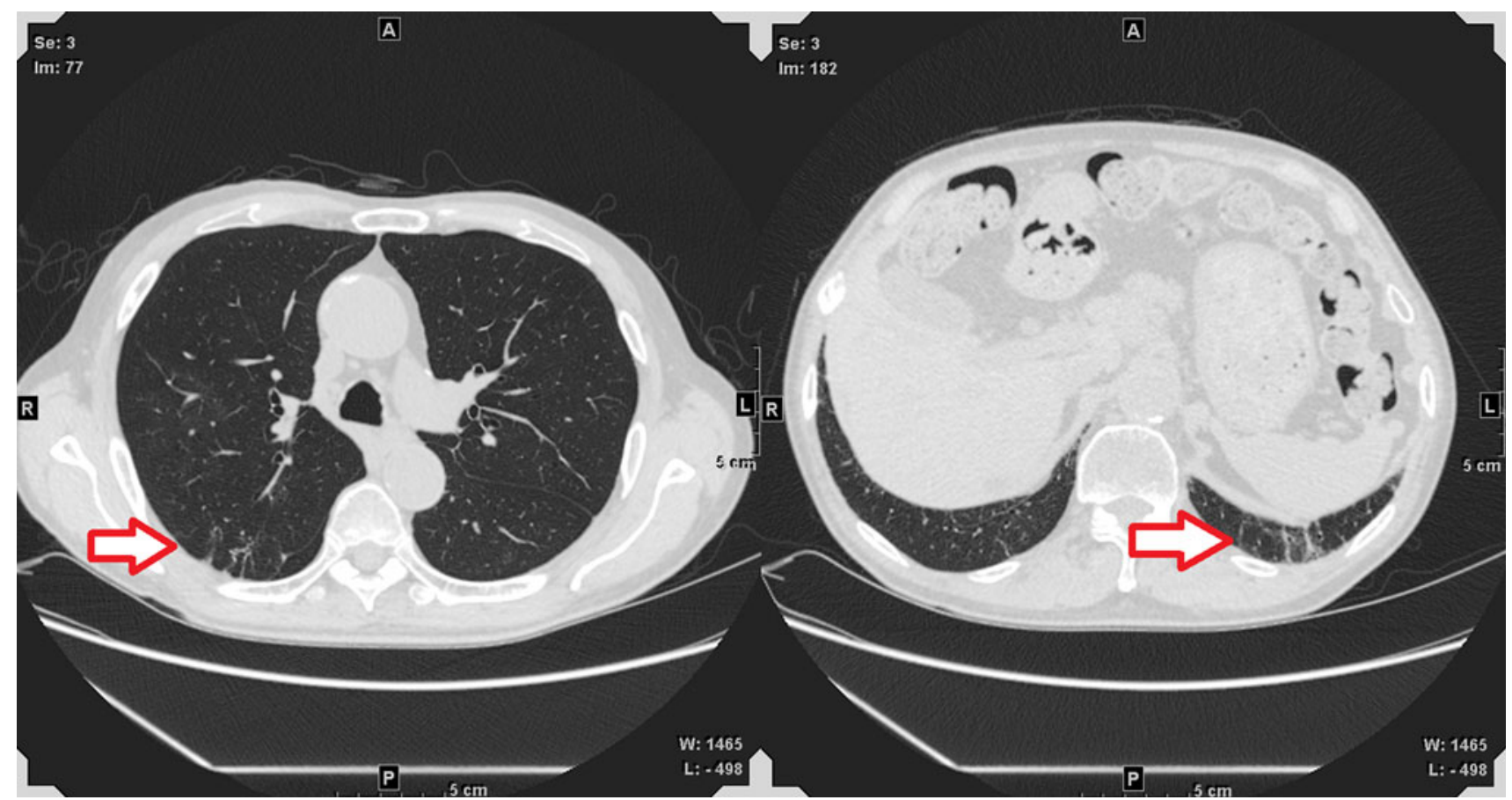

Fig. 3 Chest computed tomography image after 2 courses of gemcitabine monotherapy for metastatic urothelial cancer. A computed tomography examination revealed complete response of the lung metastases (arrow)

GEM and cisplatin (GC) combination therapy was performed. The treatment schedule was $1000 \mathrm{mg} / \mathrm{m}^{2}$ GEM on days 1,8 , and 15 , and a $75 \%$ dose of $70 \mathrm{mg} / \mathrm{m}^{2}$ cisplatin on day 2 (measured creatinine clearance rate; $47.2 \mathrm{ml} / \mathrm{min}$ ) [7]. No major side effect was observed during the GC course; however, he complained of gastrointestinal side effects such as nausea and requested the GC chemotherapy be discontinued.

The patient was switched from GC to GEM monotherapy (GEM at $1000 \mathrm{mg} / \mathrm{m}^{2}$ on days 1,8 , and 15 , and then every 28 days for 2 more courses). At a 3-month follow-up, the patient showed a complete response to the lung metastasis (Fig. 3) and a reduction in size of the L5 vertebral body metastasis (Fig. 4). He declined additional chemotherapy. Therefore, the patient was followed closely without undertaking any other therapy. At a 2-year followup after EBRT, no deterioration in the L5 vertebral body metastasis was observed.

The patient's general condition was good until he noticed a penile nodule 2.5 years after the EBRT. Physical examination revealed a nonpainful, firm, nontender nodule measuring $3.0 \mathrm{~cm}$ in size within the penis without ulceration of the overlying skin. The patient had no overt symptoms. An MRI examination revealed a cavernosum penile metastasis $3.5 \mathrm{~cm}$ in diameter (Fig. 5). Pathological examination of a needle biopsy of the nodule confirmed the lesion to be an UC that was morphologically identical to that of the bladder tumor (Fig. 6).
The patient received a single course of GEM monotherapy at this time and responded completely to the treatment for the penile metastasis (Fig. 7). At a 1-year follow-up after the GEM chemotherapy, he was still alive without any tumor metastasis.

\section{Discussion}

Recently, GC regimens have become accepted as a standard of care for advanced UC based on a survival benefit similar to that of the M-VAC regimen, with fewer adverse events [8]. However, some patients are not eligible for platinum-based regimens because of their advanced age, poor renal function, or perceived inability to tolerate the treatment $[9,10]$. Our patient did not want to continue GC chemotherapy because of the gastrointestinal side effects, such as nausea, that he experienced. In this case, the possibility that one shot of cisplatin might have had an effect on reducing the lung or bone metastasis cannot be denied. However, GEM monotherapy was highly effective against the penile tumor in this patient; therefore, we suggest that GEM played an important role in reducing the distant metastasis. Several studies have shown that in terms of tolerance and efficacy, GEM monotherapy is acceptable in elderly patients with other cancers (non-small cell lung cancer and pancreatic cancer) who are in good physical condition, with results similar to those achievable in 


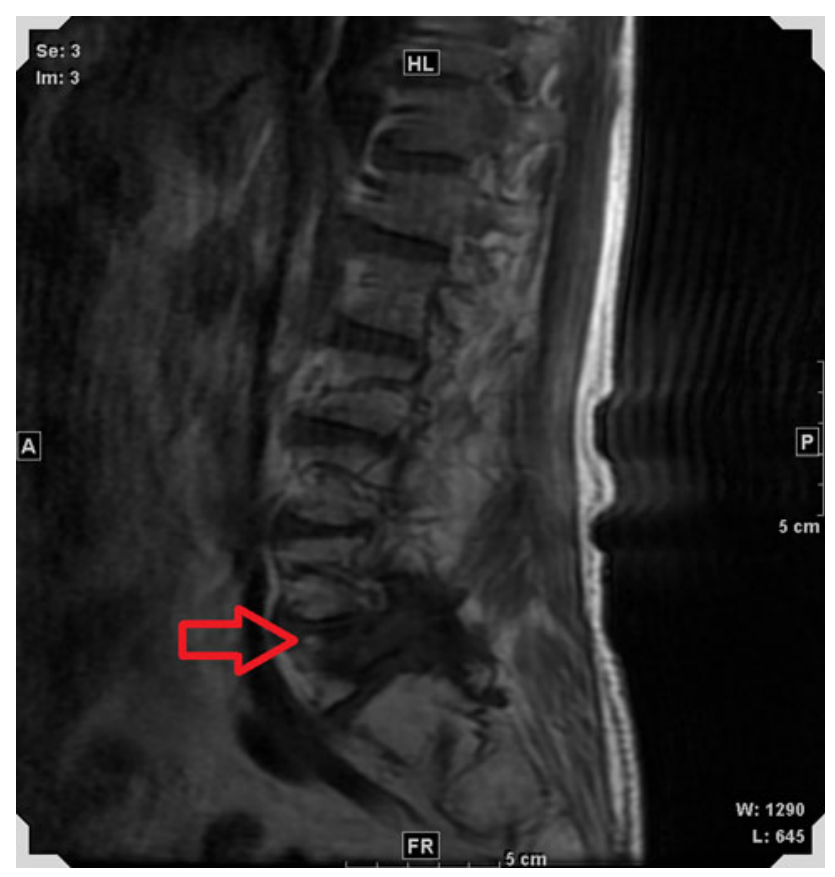

Fig. 4 Magnetic resonance image of lumbar vertebral body after 2 courses of gemcitabine monotherapy for metastatic urothelial cancer. Magnetic resonance imaging revealed a reduction in the size of the L5 vertebral body metastasis (arrow)

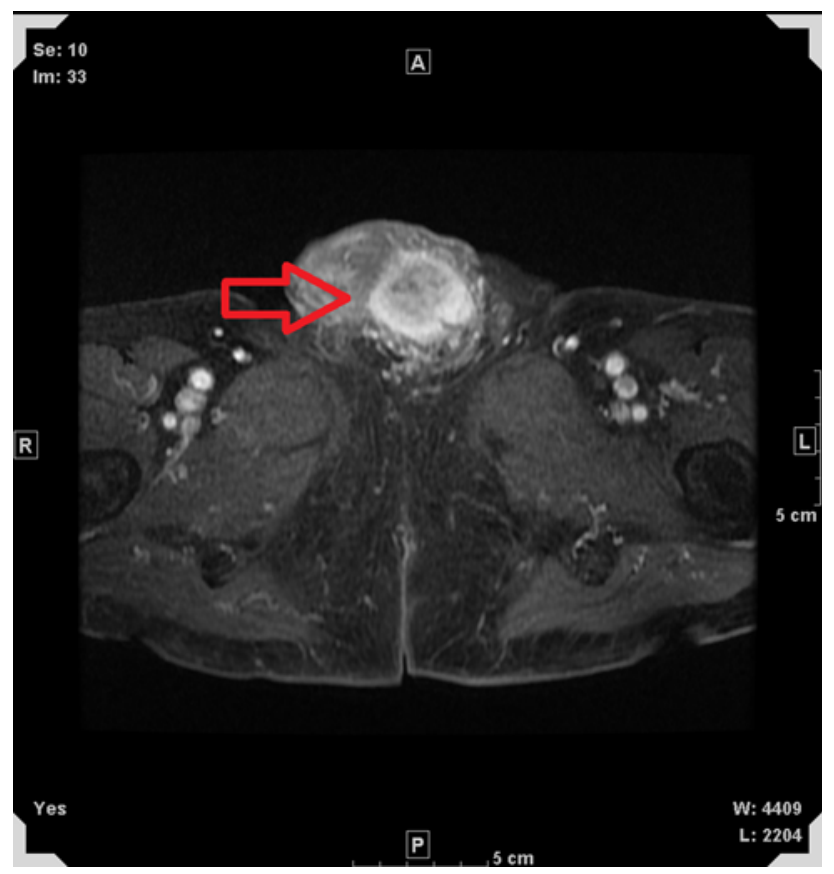

Fig. 5 Magnetic resonance image of the penile body 2.5 years after radiotherapy. Magnetic resonance imaging revealed a cavernosum penis metastasis $(3.5 \mathrm{~cm}$ in diameter, arrow)

younger patients [11-13]. Regarding UC, the efficacy and safety of GEM monotherapy in elderly patients have not been fully evaluated, and a standard of care for elderly

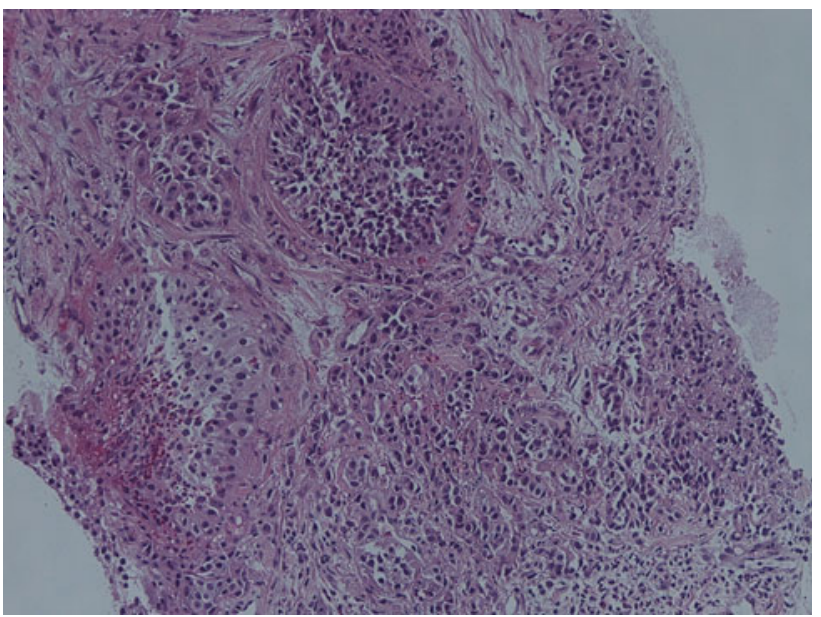

Fig. 6 Histopathological examination of a biopsy specimen of the penile tumor $(\times 100)$. Histological examination revealed a poorly differentiated metastasis from a previously diagnosed bladder urothelial carcinoma that had infiltrated the corpus cavernosum

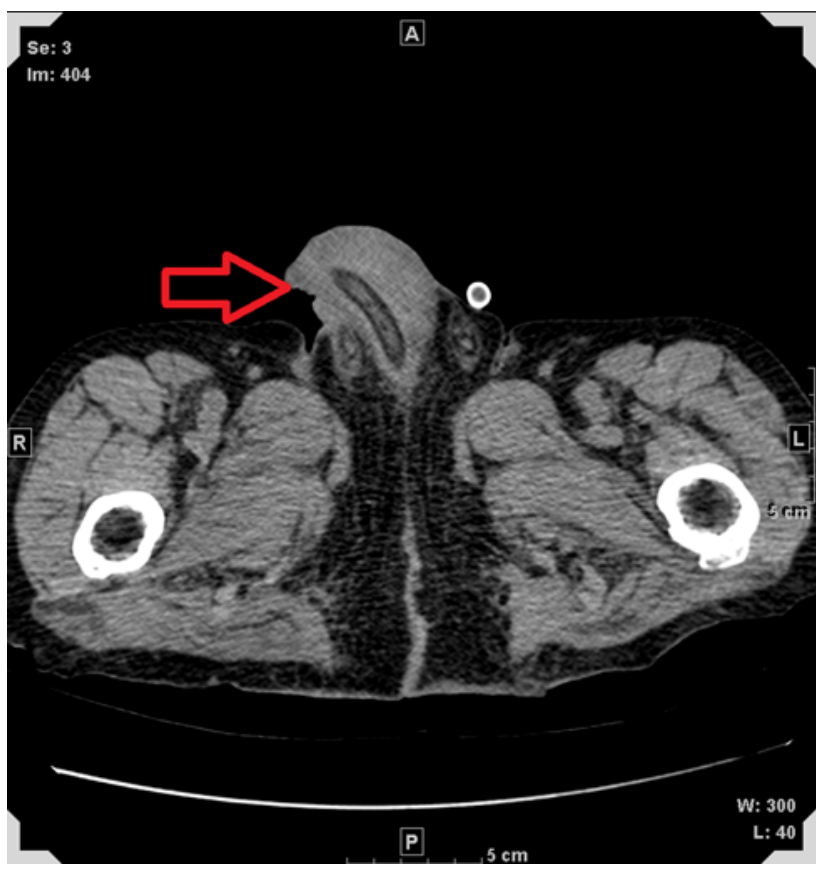

Fig. 7 Computed tomography image of the penile body after one course of gemcitabine monotherapy. A computed tomography examination revealed complete response to the penile metastasis

patients with metastatic UC has not yet been fully established. In Japan, Akaza et al. [14] evaluated GEM monotherapy in patients with advanced UC who were previously treated with a platinum-based regimen. GEM $\left(1000 \mathrm{mg} / \mathrm{m}^{2}\right.$ on days $1,8,15)$ was administered intravenously over a 28 -day period (1 cycle). This cycle was repeated at least three times or until disease progression or an intolerable adverse event was observed. In their study, 44 patients 
(age $\geq 65$ years: 23 cases; age $<65$ years: 21 cases) received GEM monotherapy, and the overall response rate was $25 \%$. The 1-year survival rate was $52.3 \%$, the median survival time was 12.6 months, and the median progression-free survival period was 3.1 months. The grade $3 / 4$ hematological toxicities were neutropenia $(47.7 \%)$, anemia $(11.4 \%)$, and thrombocytopenia $(6.8 \%)$. The major grade 3/4 non-hematological toxicity was anorexia $(9.1 \%)$. These results showed that GEM monotherapy is a sufficiently active and well-tolerated therapy for patients with metastatic UC. However, no patient over 75 years of age was included in their study. Therefore, the efficacy and safety of GEM monotherapy in elderly patients over 75-years-old with advanced UC could not be determined.

NCCN Clinical Practice Guidelines in Oncology for Senior Adult Oncology state that advanced age alone should not be an exclusion criteria for decisions regarding systemic chemotherapy in the elderly [15]. Several retrospective studies have suggested that the toxicity of chemotherapy is not as severe or as prolonged in persons older than 70 years as once expected [16, 17]. However, the results of these studies cannot be generalized for the following reasons. First, only a few patients were 80 or older; therefore, minimal information is available for the oldest patients. Second, the older patients involved in these studies were not representative of the general older population, because their inclusion criteria were strict and they were probably healthier than most older patients. Therefore, appropriate patient selection is extremely important to maximize the benefits and minimize the side effects of systemic chemotherapy in older patients.

Gemcitabine has fewer related severe adverse events than other cytotoxic drugs. The major known adverse events of GEM are neutropenia, thrombocytopenia, and nausea/vomiting [14]. Therefore, GEM monotherapy is recommended for patients aged 70 years or more when their performance status is good. However, adverse effects, especially hematological toxicity, must be monitored more carefully during the first course of treatment in elderly patients, and continuing the therapy while reducing the dose of GEM may bring about better results if hematological toxicity has occurred [11].

Penile metastases from primary UC of the urinary system are extremely rare. To date, only about 370 cases of penile metastases have been reported [4]. The primary origins of these metastases include the urinary bladder in $30-35 \%$, the prostate in $30 \%$, the rectosigmoid colon in $13 \%$, the kidneys in $8-10 \%$, and the testes in $5 \%$ [18]. Penile metastases present as a nonulcerative nodule in $51 \%$ of the cases. Other manifestations of penile metastases include priapism in $27 \%$, urinary symptoms (urethral hemorrhage, hematuria, incontinence, and irritative and obstructive symptoms) in $27 \%$, pain in $17 \%$, retention in
$13 \%$, and skin lesions in $11 \%$ [19]. In the present case, the patient had a nonulcerative penile nodule without any overt symptoms. Penile metastases of UC are usually diagnosed by biopsy, and non-invasive methods, such as MRI or color-coded duplex ultrasonography, may be used for disease staging [20]. The optimal treatment for penile metastases requires a multidisciplinary approach that is correlated with the disease extent. Treatment options in these patients include local excision, partial or total penectomy, EBRT, and chemotherapy.

The present patient had a complete response to the GEM monotherapy in the penis and lung metastases, and a significant reduction in the size of the L5 vertebral body metastasis. This unusual case suggests that patients with metastatic UC may undergo GEM monotherapy even if they are 80 or older. The risk benefit ratio of this treatment should be discussed in a multidisciplinary context, and these patients should actively participate in therapeutic decisions.

Conflict of interest The authors declare that they have no conflict of interest.

\section{References}

1. Marugame T, Kamo K, Katanoda K et al (2006) Cancer incidence and incidence rates in Japan in 2000: estimates based on data from 11 population-based cancer registries. Jpn J Clin Oncol 36:668-675

2. Shelley M, Cleves A, Wilt TJ et al (2011) Gemcitabine for unresectable, locally advanced or metastatic bladder cancer. Cochrane Database Syst Rev 13:CD008976

3. Hizli F, Berkmen F (2006) Penile metastasis from other malignancies. A study of ten cases and review of the literature. Urol Int 76:118-121

4. Cherian J, Rajan S, Thwaini A et al (2006) Secondary penile tumours revisited. Int Semin Surg Oncol 3:33

5. Akman Y, Cam K, Kavak A et al (2003) Extensive cutaneous metastasis of transitional cell carcinoma of the bladder. Int J Urol 10:103-104

6. Bordeau KP, Lynch DF (2004) Transitional cell carcinoma of the bladder metastatic to the penis. Urology 63:981-983

7. Kintzel PE, Dorr RT (1995) Anticancer drug renal toxicity and elimination: dosing guidelines for altered renal function. Cancer Treat Rev 21:33-64

8. von der Maase H, Sengelov L, Roberts JT et al (2005) Long-term survival results of a randomized trial comparing gemcitabine plus cisplatin, with methotrexate, vinblastine, doxorubicin, plus cisplatin in patients with bladder cancer. J Clin Oncol 23:4602-4608

9. Galsky MD, Hahn NM, Rosenberg J et al (2011) Treatment of patients with metastatic urothelial cancer "Unfit" For cisplatinbased chemotherapy. J Clin Oncol 29:2432-2438

10. Linardou H, Aravantinos G, Efstathiou E et al (2004) Gemcitabine and carboplatin combination as first-line treatment in elderly patients and those unfit for cisplatin-based chemotherapy with advanced bladder carcinoma: phase II study of the Hellenic cooperative oncology group. Urology 64:479-484

11. Yamagishi Y, Higuchi H, Izumiya M et al (2010) Gemcitabine as first-line chemotherapy in elderly patients with unresectable pancreatic carcinoma. J Gastroenterol 45:1146-1154 
12. Shepherd FA, Abratt RP, Anderson H et al (1997) Gemcitabine in the treatment of elderly patients with advanced non-small cell lung cancer. Semin Oncol 24:S7-50-S7-55

13. Marechal R, Demols A, Gay F et al (2008) Tolerance and efficacy of gemcitabine and gemcitabine-based regimens in elderly patients with advanced pancreatic cancer. Pancreas 36:e16-e21

14. Akaza H, Naito S, Usami M et al (2007) Efficacy and safety of gemcitabine monotherapy in patients with transitional cell carcinoma after cisplatin-containing therapy: a Japanese experience. Jpn J Clin Oncol 37:201-206

15. Hurria A, Browner IS, Cohen HJ et al (2012) Senior adult oncology. J Natl Compr Canc Netw 10:162-209

16. Ibrahim NK, Frye DK, Buzdar AU et al (1996) Doxorubicinbased chemotherapy in elderly patients with metastatic breast cancer. Tolerance and outcome. Arch Intern Med 156:882-888
17. Lichtman SM, Wildiers $\mathrm{H}$, Chatelut $\mathrm{E}$ et al (2007) International society of geriatric oncology chemotherapy taskforce: evaluation of chemotherapy in older patients - an analysis of the medical literature. J Clin Oncol 25:1832-1843

18. Berger AP, Rogatsch H, Hoeltl L et al (2003) Late penile metastasis from primary bladder carcinoma. Urology 62:145

19. Chan PT, Begin LR, Arnold D et al (1998) Priapism secondary to penile metastasis: a report of two cases and a review of the literature. J Surg Oncol 68:51-59

20. Sonmez NC, Coskun B, Arisan S et al (2009) Early penile metastasis from primary bladder cancer as the first systemic manifestation: a case report. Cases J 2:7281 